\title{
Tunneled Pleural Catheters in the Management of Chylothorax from Central Venous Catheter-Related Superior Vena Cava Obstruction
}

\author{
Vanessa Luks ${ }^{\mathrm{a}}$ Ahmed Aljohaney $^{\mathrm{b}}$ Kayvan Amjadi $^{\mathrm{a}}$ \\ a University of Ottawa, Ottawa, Ont., Canada; ${ }^{b}$ Faculty of Medicine, King Abdulaziz University, Jeddah, Saudi Arabia
}

\section{Established Facts}

- Pleural effusions from benign superior vena cava obstruction may be chylous, exudative, or transudative.

- Management of chylous pleural effusions includes immediate relief of dyspnea with pleural fluid evacuation, treatment of the underlying cause, and institution of conservative measures such as a mediumchain triglyceride diet or total parenteral nutrition, and octreotide. Surgical options are reserved for failure of medical therapy.

\section{Novel Insights}

- Pleural effusions from benign superior vena cava obstruction may change from one type to another depending on systemic venous hypertension, volume status, and likely other factors as well.

- Although there are concerns regarding malnutrition and/or immunosuppression following long-term drainage of chylothoraces, this report illustrates 2 cases of prolonged chylothorax drainage showing minimal patient morbidity.

\section{Key Words}

Chylothorax $\cdot$ Indwelling pleural cathether $\cdot$ Superior vena cava syndrome

\footnotetext{
Abstract

Chylothorax is characterized by the accumulation of chyle in the pleural space, of which the most common cause is trauma or neoplasm. Although chylothorax accounts for a small proportion of clinical pleural effusions, prompt recognition
}

is needed to avoid malnutrition, immunodeficiency, and fibrothorax. We report 2 patients with superior vena cava obstruction caused by tunneled venous catheters resulting in chylothorax and demonstrate the potential safety of tunneled pleural catheters for prolonged chylothorax drainage in an outpatient setting with rigorous follow-up. Changes in pleural fluid chemistries of the effusions and the possible pathophysiology were assessed with a review of the literature on pleural fluid chemistries in superior vena cava obstruction.

\section{KARGER}

E-Mail karger@karger.com www.karger.com/res
(C) 2013 S. Karger AG, Basel

0025-7931/13/0861-0067\$38.00/0
Vanessa Luks

501 Smyth Road, Box 211

Ottawa, ON K1H 8L6 (Canada)

E-Mail vanessaluks@gmail.com 


\section{Introduction}

Chylothorax refers to the accumulation of chyle in the pleural space. Chyle is a milky-appearing lymphatic fluid of intestinal origin containing high levels of fat, proteins, and immunoglobulins. Chyle is carried back into the venous system from the cisterna chyle via the thoracic duct. Obstruction or laceration of the thoracic duct leads to leakage of chyle into the pleural space. Numerous causes of chylothorax have been reported. In the literature, chylothorax due to superior vena cava obstruction (SVCO) caused by tunneled central venous catheters is rare. In the literature, 2 cases of chylothorax and chylopericardium causing cardiac tamponade in the setting of SVCO due to indwelling central venous catheters have been reported. Percutaneous transluminal angioplasty (PTA) of the SVCO was successful in both cases leading to restoration of flow and resolution of the chylothoraces $[1,2]$. Two cases of chylothorax from left-sided subclavian vein Port-A-Caths were also mentioned in a retrospective chart review [3]. These reports discuss the pathophysiology of SVCO from indwelling catheters as well as the success of endovascular therapy in relieving SVCO and associated chylothoraces. Unique points for discussion in our cases include the changes in the biochemistry of the effusions in the 2nd case, and the longterm management with indwelling pleural catheters in both cases, which historically has been strongly discouraged.

\section{Case 1}

Case 1, a 62-year-old female, presented with a 3-month history of progressive dyspnea, orthopnea, facial swelling, and new headaches. Her past medical history was remarkable for stage 3 serous papillary adenocarcinoma of the ovary diagnosed in 1990 and treated with surgical debulking and chemotherapy. In 1998, 10 years before presenting with SVCO and chylothorax, she developed metastases of the liver and central tendon of the right diaphragm which were treated with chemotherapy via a new right subclavian Port-ACath (Smiths Medical). This was followed by wedge resection of the involved liver and removal of the central tendon of the right diaphragm and its repair using polytetrafluoroethylene. Ten years later, physical examination revealed facial swelling, dilated neck and chest wall veins, and decreased breath sounds over the right hemithorax. Her chest X-ray demonstrated a large right pleural effusion; the right Port-A-Cath from 1998 was still in place. Diagnostic thoracentesis yielded milky fluid, which retained this appearance after standing for $43 \mathrm{~h}$ in the laboratory refrigerator. Pleural fluid was consistent with a chylothorax: triglycerides $11.7 \mathrm{mmol} / \mathrm{l}$ (pH: 7.5, glucose: 5.6 mmol/l, LDH: $105 \mathrm{U} / \mathrm{l}$, protein: $36 \mathrm{~g} / \mathrm{l}$, and cholesterol: $1.7 \mathrm{mmol} / \mathrm{l}$; serum testing was not available). Repeated cytological examinations were reported as chronic inflammation and microbiological cultures were negative. CA-125 was elevated at $244 \mathrm{kU} / \mathrm{l}$; however, there was no radiologic evidence of cancer recurrence, and subse-

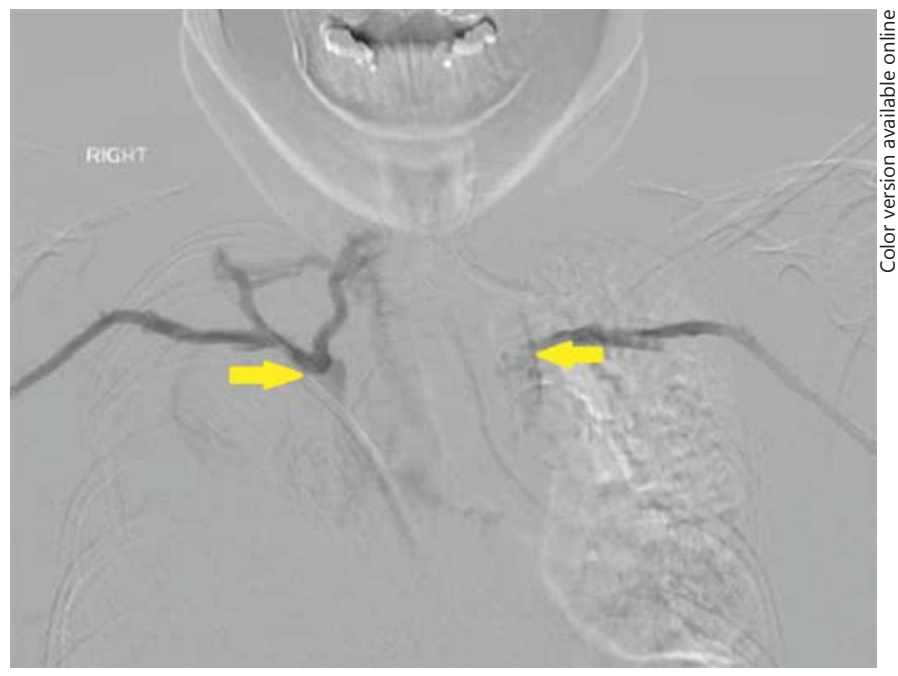

Fig. 1. Bilateral venogram demonstrating bilateral occlusion of the brachiocephalic veins (arrows) and collateral pathways via the external jugular and anterior jugular veins.

quently there was a spontaneous decline in the CA-125 level. A mammogram showed a right breast nodule but biopsy confirmed this to be a benign phyllodes tumor. The possibility that the chylothorax resulted from surgical disruption of the thoracic duct during diaphragmatic resection was considered less likely due to the long time interval between surgery and her presentation. Most postoperative traumatic chylothoraces manifest within 10 days of the culprit procedure. A contrast-enhanced CT scan of the thorax demonstrated marked collateral venous circulation at the level of the mediastinum with poor opacification of the superior vena cava suggestive of chronic SVCO. Angioplasty was planned but attempts to cross the superior vena cava occlusion either from above via the Port-A-Cath or from below via a femoral approach were unsuccessful (fig. 1). The Port-A-Cath was explanted and the patient was treated with long-term anticoagulation for chronic catheter-associated venous thromboembolism causing SVCO. A PleurX (CareFusion), an indwelling tunneled pleural catheter (TPC), was inserted for ongoing drainage of the chylothorax. She continued to drain about 500 $\mathrm{ml}$ of chyle 3 times a week despite instituting a medium-chain triglyceride dietary regimen. A 17-day trial of total parenteral nutrition failed to reduce the rate of drainage. Therapeutic surgical ligation of the thoracic duct was offered and declined by the patient. She was then followed up on a regular basis. Drainage of the chylothorax ceased 9 months after TPC insertion, with chest CT showing a tiny loculated right pleural effusion. At this point, the TPC was removed. Subsequently, she was followed up radiographically for 2.5 years. The tiny right loculated effusion diminished further, and there was some residual right pleural thickening.

\section{Case 2}

Case 2, a 63-year-old female, presented with a 1-month history of progressive dyspnea. Her past medical history included ischemic nephropathy on home hemodialysis since 2006, severe 

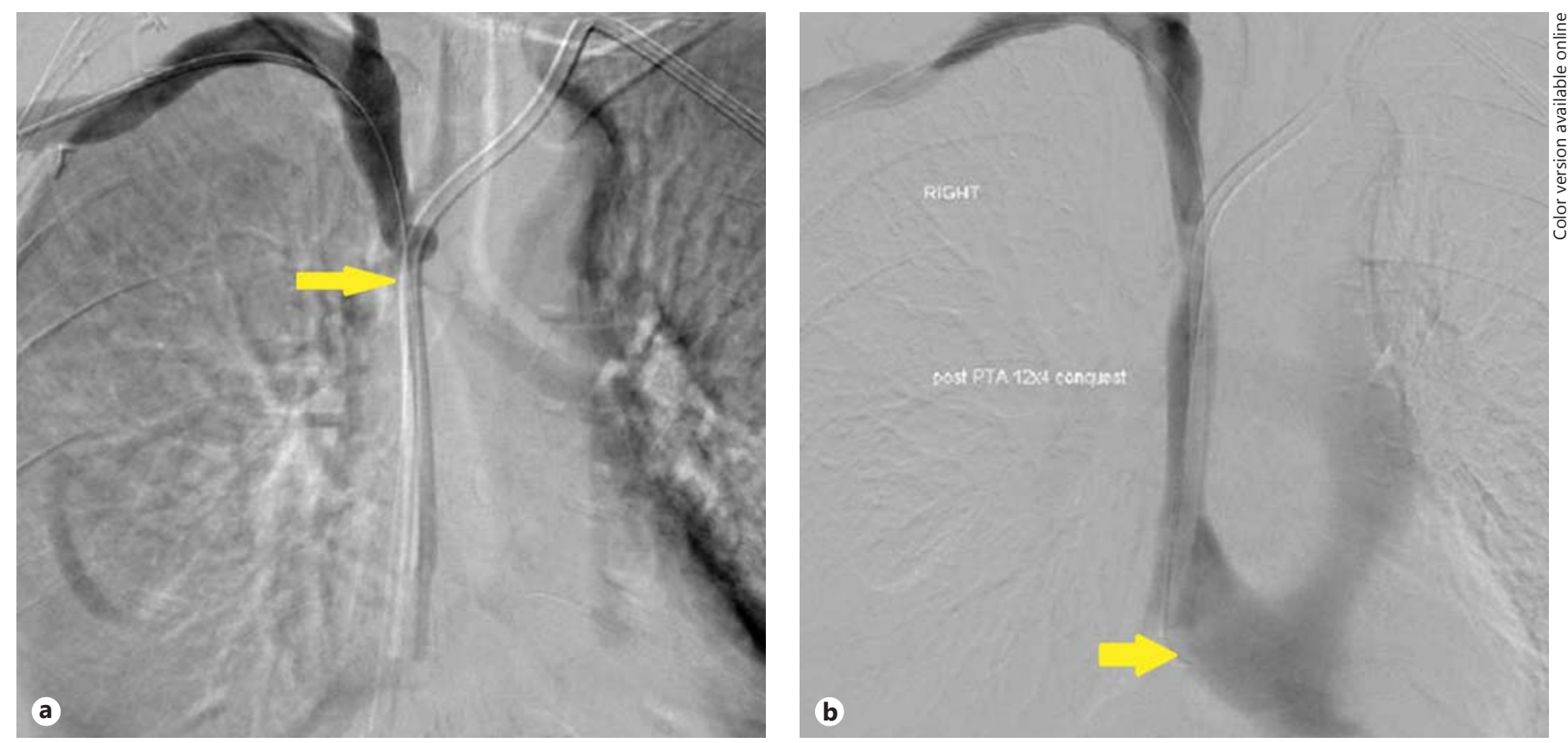

Fig. 2. a Digital subtraction angiography through right brachial basilic transposition fistula showing SVCO. b Postpercutaneous angioplasty showing restoration of SVCO patency with flow into the right atrium (arrow).

peripheral arterial disease, multiple sclerosis, essential thrombocytosis with a positive JAK-2 mutation, and recurrent central vein stenoses/thromboses requiring chronic anticoagulation with warfarin. She had a left-sided Hickman catheter (BARD) inserted at the time of commencing hemodialysis (1 year prior to her presentation with SVCO and chylothorax). Physical examination revealed reduced breath sounds and dullness to percussion over both hemithoraces. A CT scan of the thorax confirmed bilateral pleural effusions (larger on the left side) and a small pericardial effusion. Thoracentesis from the left side yielded milky pleural fluid, which was confirmed to be a chylothorax: triglycerides 12.99 $\mathrm{mmol} / \mathrm{l}$, chylomicrons present [pH 7.6, glucose $6.1 \mathrm{mmol} / \mathrm{l}, \mathrm{LDH}$ $116 \mathrm{U} / \mathrm{l}$, protein $47 \mathrm{~g} / \mathrm{l}$, serum protein $74 \mathrm{~g} / \mathrm{l}$, serum LDH $245 \mathrm{U} / \mathrm{l}$ (upper limit of normal serum LDH is $192 \mathrm{U} / \mathrm{l}$ at our institution), and cholesterol $1.1 \mathrm{mmol} / \mathrm{l}$. Pleural fluid cytology showed lymphocytes and reactive mesothelial cells; microbiological cultures were negative. Thoracentesis on the right side was also consistent with a chylothorax: triglycerides $2.70 \mathrm{mmol} / \mathrm{l}$ ( $\mathrm{pH} 7.6$, glucose 6.0 $\mathrm{mmol} / \mathrm{l}, \mathrm{LDH} 114 \mathrm{U} / \mathrm{l}$, and protein $29 \mathrm{~g} / \mathrm{l}$; concurrent serum values were not available). We anticipated the need for long-term pleural drainage and therefore a TPC was used for drainage of the larger left pleural effusion. The patient was also placed on a mediumchain triglyceride diet, but the quantity of drainage did not diminish, and the patient refused total parenteral nutrition. A rightsided TPC was eventually also inserted to relieve dyspnea associated with reaccumulation of the large right chylous effusion. A workup for tuberculosis and lymphoma was negative. To further investigate the cause of chylothorax, a central venogram was performed and showed new onset SVCO, likely aggravated by the Hickman catheter. This was followed by superior vena cava dilata- tion using a high-pressure $12-\mathrm{mm}$ balloon with prolonged inflation time resulting in restoration of patency of the superior vena cava (fig. 2). After angioplasty of the SVCO, the pleural fluid became transudative bilaterally. Two months later, upon resampling of both effusions, they were both exudative. Five months after insertion of the left TPC and 2 months after insertion of the right TPC, both catheters were removed as there was no further significant fluid drainage and chest X-ray confirmed no reaccumulation. Subsequent to TPC removal, there was intermittent minimal reaccumulation of the effusions which would resolve, along with her dyspnea, with repeat angioplasty of recurrent SVCO. She was assessed by thoracic surgery who suggested repeat angioplasty rather than thoracic duct ligation given the uncertainty of success and her high perioperative risk. She eventually had a superior vena cava stent successfully placed for the recurrent SVCO. Radiographic follow-up 4.5 years following TPC removal showed that very small effusions, mostly on the right side and rarely bilaterally, accumulate and resolve following repeat angioplasty of various stenotic segments of the superior vena cava.

\section{Discussion}

Pleural effusions associated with SVCO can take one of three forms: chylous, transudative nonchylous, or exudative nonchylous. The reason for adding 'nonchylous' to descriptions of pleural fluid in the last sentence is that chylous effusions themselves may be transudative or exu- 
dative $[4,5]$. In a retrospective study over 6 years, Rice et al. [6] found 52 cases of pleural effusions secondary to SVCO. Although most of the pleural effusions associated with benign SVCO were not sampled, those that were sampled were mostly exudative ( 4 of 5 ) and/or chylous (2 of 5) in nature [6]. In our 2nd case, bilateral chylothoraces (chylous exudative) changed to nonchylous transudative effusions a few days after PTA of the SVCO, and a couple of months later were found to be nonchylous exudates. In the setting of SVCO, chylothorax is presumed to occur due to high venous pressure distal to the obstruction which then increases the pressure within the thoracic duct causing chyle leakage. Given the resolution of the SVCO-related chylous effusions in the literature after angioplasty or stenting, thoracic duct disruption is felt to be unlikely [6]. Transudative effusions due to SVCO are hypothesized to occur because of increased systemic venous hydrostatic pressure that impedes lymphatic drainage from the pleural cavities [7]. The documented change in pleural chemistry from chylothorax to transudative effusion after PTA of the SVCO in our 2nd case is likely because the central venous pressure dropped sufficiently after the procedure to resolve the thoracic duct hypertension and the chyle leakage, but not sufficiently, at least within the first few days, to provide adequate lymphatic drainage via the parietal pleura. In the study by Rice et al. [6], none of their patients with intravascular device-associated SVCO had transudative effusions; however, data on changes in pleural fluid after therapy were not provided, and the majority of the patients in their series with benign effusions did not undergo pleural fluid sampling at any point. The etiology of exudative effusions from benign SVCO is even more obscure. Rice et al. [6] hypothesized the coexistence of central venous catheter-derived pulmonary emboli that may cause an exudative effusion, incorrect classification of pleural effusions as exudative as a result of the highly sensitive Light criteria, or changes in fluid biochemistry following treatment with diuretics [3]. In our 2nd case, possible explanations for the change from transudative to exudative effusion include changes in the volume status with aggressive fluid removal in dialysis, a similar phenomenon to the effect of acute diuresis on pleural fluid chemistry, or, less likely, inflammation from the indwelling pleural catheters. Venous thromboembolism seems unlikely given that case 2 was on treatment with warfarin at therapeutic levels long before SVCO occurred. In the 1st case, the pleural effusion was sampled only once and was found to be chylous.

Regarding the management of chylothorax, the first principle is pleural space drainage for acute symptomatic relief. The next steps are aimed at decreasing the flow of chyle in the thoracic duct to permit healing, and of course treatment of the underlying cause. To accomplish the former, patients are changed to a medium-chain triglyceride diet (these fats are absorbed directly into the portal system, bypassing the intestinal lymphatic system) or, if this is unsuccessful, bowel rest with total parenteral nutrition. The addition of octreotide can also decrease intestinal lymph production $[8,9]$. Surgical options are reserved for failure of conservative management. Typically, thoracic duct ligation is first considered, but talc pleurodesis, pleurectomy, or pleuroperitoneal shunts are further options in case thoracic duct ligation fails [9]. Talc pleurodesis has been reported to be successful [10], but excessive inflammation and acute respiratory distress syndrome may also be encountered, particularly with small particle talc [11, 12].

Both of our cases had symptomatic relief with initial drainage and either refused or had no improvement, at least in quantity of drainage, with dietary support. Regarding the underlying cause of SVCO, PTA is recommended for symptomatic patients with benign SVCO, and stenting is recommended for recurrent stenosis. In our 2nd case, PTA of SVCO, and eventual stenting because of recurrence, resulted in resolution of the chylothorax (though repeat angioplasty procedures were required even after stenting). In the 1st case, as angioplasty was not successful, the likely cause of resolution was a combination of spontaneous pleurodesis from the indwelling catheter (as evidenced by loculation and pleural thickening seen on chest CT), and perhaps further development of collaterals to decrease the high venous pressure at the outflow of the thoracic duct into the superior vena cava (as evidenced by slightly larger and more numerous venous collaterals on chest CT at the time of TPC removal compared to the time of TPC insertion). Avoiding prolonged drainage of the chylothorax is recommended to prevent malnutrition and immunosuppression due to loss of immunoglobulins, fat, proteins, and electrolytes contained in the chyle. Unlike the prior case reports in which pleural fluid drainage was restricted to 7-11 days, both of our cases underwent prolonged chylothorax drainage for 5-9 months while we tried to address the underlying cause. The role of TPC to provide interrupted drainage for patients with recurrent malignant pleural effusion is well documented $[13,14]$. The current literature lacks sufficient evidence to recommend TPC use in nonmalignant pleural disease such as chylothorax [15]. Nevertheless, we elected to use this drainage modality in both cases as alternative options were quite limited 
and we anticipated the need for long-term drainage for symptom relief. The 1st patient refused to undergo thoracic ligation and the 2 nd patient had significant comorbidities. Regular follow-up visits and monitoring were provided through our chronic pleural effusion outpatient clinic. This is a designated clinic to provide comprehensive care for patients with chronic pleural effusions treated with TPC [16]. Neither patient developed clinically overt malnutrition or infection despite declines in albumin and lymphocyte counts. With respect to the first case, lymphocyte counts and albumin levels before TPC insertion were $1.0 \times 10^{9} / 1$ and $36 \mathrm{~g} / \mathrm{l}$, respectively, and then ranged between 0.5 and $0.7 \times 10^{9} / 1$ for lymphocytes, and between 24 and $29 \mathrm{~g} / \mathrm{l}$ for albumin (normal values for lymphocytes are $1.0-1.4 \times 10^{9} / 1$ and for albumin $35-48$ $\mathrm{g} / \mathrm{l}$, respectively, at our institution) throughout the drainage period. In the 2 nd case, lymphocyte counts and albumin levels before the procedure were $1.3 \times 10^{9} / 1$ and 39 $\mathrm{g} / \mathrm{l}$, respectively. Lymphocyte counts ranged between 0.7 and $0.8 \times 10^{9} / 1$ throughout drainage, and albumin levels between 32 and $34 \mathrm{~g} / \mathrm{l}$. There was a single nadir in albu$\min (19 \mathrm{~g} / \mathrm{l})$, but this was concurrent with urinary tract infection with Escherichia coli and Proteus mirabilis, which was associated with significant serum leukocytosis. One month after TPC removal, albumin increased to 39 $\mathrm{g} / \mathrm{l}$ and lymphocytes to $0.9 \times 10^{9} / \mathrm{l}$ in case 2 (similar data are not available for case 1). A case report on the use of TPC for cancer-related chylothorax compared to controls (controls received repeated therapeutic thoracenteses, talc pleurodesis, or pleuroperitoneal shunting) showed no difference in the drop in albumin or lymphocytes between the TPC and control groups, with the added benefit of decreased risk of additional procedures after the index procedure in the TPC group [17]. In conclusion, this report illustrates 2 cases of nonmalignant chylothorax treated with TPC for prolonged drainage under rigorous monitoring with minimal patient morbidity.

\section{References}

1 Veroux P, Veroux M, Bonanno MG, Tumminelli MG, Baggio E, Petrillo G: Long-term success of endovascular treatment of benign superior vena cava occlusion with chylothorax and chylopericardium. Eur Radiol 2002; 12:S181-S184.

$\checkmark 2$ Adekile A, Adeogoroye A, Tedla F, Levin D, Salifu MO: Chylothorax and chylopericardial tamponade in a hemodialysis patient with catheter-induced superior vena cava stenosis. Semin Dial 2009;22:576-579.

$>3$ Rice TW: Pleural effusions in superior vena cava syndrome: prevalence, characteristics and proposed pathophysiology. Curr Opin Pulm Med 2007; 13:324-327.

4 Skouras V, Kalomenidis I: Chylothorax: diagnostic approach. Curr Opin Pulm Med 2010; 104:1-8.

5 Ryu JH, Tomassetti S, Maldonado F: Update on uncommon pleural effusions. Respirology 2011;16:238-243.
6 Rice TW, Rodriguez RM, Barnette R, et al: Prevalence and characteristics of pleural effusions in superior vena cava syndrome. Respirology 2006;11:299-305.

7 Diaz-Guzman E, Culver DA, Stoller JK: Transudative chylothorax: report of two cases and review of the literature. Lung 2005;183:169175.

$>8$ Kalomenidis I: Chylothorax and octreotide. Curr Opin Pulm Med 2006;12:264-267.

$>9$ McGrath EE, Blades Z, Anderson PB: Chylothorax: aetiology, diagnosis, and therapeutic options. Respir Med 2010;104:1-8.

10 Mares DC, Mathur PN: Medical thoracoscopic talc pleurodesis for chylothorax due to lymphoma: a case series. Chest 1998;114:731735.

11 Dressler CM, Olak J, Herndon JE II, et al: Phase III intergroup study of talc poudrage versus talc slurry sclerosis for malignant pleural effusion. Chest 2005;127:909-915.

-12 Arellano-Ordon E, Romero FA, Juan JM: Small particle-size talc is associated with poor outcome and increased inflammation in thoracoscopic pleurodesis. Respiration 2012, DOI: $10.1159 / 000342042$.
13 Van Meter ME, McKee KY, Kohlwes RJ: Efficacy and safety of tunneled pleural catheters in adults with malignant pleural effusions: a systematic review. J Gen Intern Med 2011;26: 70-76.

14 Sabur N, Chee A, Stather DA: The impact of tunnelled pleural catheters on quality of life of patients with malignant pleural effusions. Respiration 2013;85:36-42.

15 Chee A, Tremblay A: The use of tunneled pleural catheters in the treatment of pleural effusions. Curr Opin Pulm Med 2011;17:237241.

16 Graver A, Amjadi K: Outpatient management of malignant pleural effusions: the Ottawa experience. Ontario Thorac Rev 2010;22:3-11.

-17 Jimenez CA, Mahtre AD, Martinez CH, Eapen GA, Onn A, Morice RC: Use of an indwelling pleural catheter for the management of recurrent chylothorax in patients with cancer. Chest 2007;132:1584-1590. 